\title{
Se former à l'usage des dispositifs de téléprésence, visioconférence et webconférence en enseignement supérieur États des lieux dans deux universités
}

Self-training with the use of telepresence, videoconferencing and web conferencing devices in higher education: State of play in two universities

\section{Formarse para saber utilizar dispositivos de telepresencia, videoconferencia y webconferencia en educación superior: Estado de la cuestión en dos universidades}

Simon Bolduc, doctorant en pédagogie de l'enseignement supérieur

Université de Sherbrooke, Canada

simon.bolduc@usherbrooke.ca

Matthieu Petit, professeur

Université de Sherbrooke, Canada

matthieu.petit@usherbrooke.ca

Florian Meyer, professeur

Université de Sherbrooke, Canada

florian.meyer@usherbrooke.ca

Denis Bédard, professeur

Université de Sherbrooke, Canada

denis.bedard@usherbrooke.ca

Rana Challah, Enseignante, chercheure-postdoctorante

Université Rennes 2, France

ranachallah@gmail.com 


\section{RÉSUMÉ}

Les dispositifs technopédagogiques de type visioconférence sont des technologies émergentes de plus en plus utilisées en enseignement supérieur. Certains établissements d'enseignement investissent largement dans leur implantation et dans leur déploiement au service de la formation à distance. Mais qu'en est-il des usages qu'en font les formateurs? Qu'en est-il du rôle des conseillers/ingénieurs pédagogiques dans un tel contexte? Le présent article traite d'une étude réalisée dans le cadre du projet TOPIC (Téléprésence comme OPportunité d'Innovation dans la Conception de formation) tiré d'un travail conjoint entre l'Université de Sherbrooke (Canada) et l'Université Bretagne Loire (France). II s'agit d'une enquête en ligne réalisée auprès de 174 formateurs des deux universités partenaires pour répondre à la question : "Quels sont les usages actuels, le niveau d'aisance et les attentes des formateurs face aux dispositifs technopédagogiques de type visioconférence?" . L'enquête fait ressortir que les dispositifs de visioconférence sont peu utilisés et que les formateurs sont peu formés à leur utilisation. Malgré tout, une majorité des répondants se dit à l'aise avec leur capacité d'enseigner avec le numérique. En termes de formations, il ressort de l'enquête que les formateurs privilégient des ressources propices à l'autoformation, à un accompagnement personnalisé ou à une combinaison de ressources et d'accompagnement.

Mots-clés : téléprésence, visioconférence, webconférence, conseiller pédagogique, formation continue, dispositif technopédagogique, enseignement supérieur, développement professionnel

\section{ABSTRACT}

Technopedagogical devices such as video conferencing are emerging technologies that are increasingly in use in higher education. Some educational institutions invest heavily in their implementation and deployment for distance learning education. However, little is known about the uses that trainers make of them and about the role of educational advisors in this context. This paper presents a study carried out as part of the TOPIC project (Téléprésence comme OPportunité d'Innovation dans la Conception de formation) a joint project between the University of Sherbrooke (Canada) and the Université Bretagne Loire (France). An online survey was sent to 174 trainers from the two partner universities using the following questions: "What are the current uses, the level of ease and the expectations of teachers vis-à-vis technopedagogical (videoconference) devices?". Survey findings show that video conferencing devices are not widely used, that there is little use of videoconferencing devices, and that trainers are poorly trained (or receive little training) to use them. Nevertheless, a majority of respondents to the online survey indicated that they were confident in their ability to teach using digital technology. In terms of training, the online survey findings show that trainers favour the use of self-training resources, personalized accompaniment support or a combination of resources and support.

Keywords: telepresence, videoconferencing, webconferencing, educational advisor, technopedagogical devices, in-service training, higher education, professional development 
Los dispositivos de tecnología pedagógica de tipo videoconferencia son tecnologías emergentes cada vez más utilizadas en educación superior. Algunas instituciones de educación realizan una gran inversión para la implantación y el desarrollo de estas tecnologías en la formación a distancia. Sin embargo, podríamos preguntarnos lo siguiente: ¿Los formadores utilizan esta tecnología como herramienta pedagógica? ¿Qué papel tienen los consejeros pedagógicos en este contexto? Este artículo trata del estudio realizado en el marco del proyecto TOPIC (Téléprésence comme OPportunité d'Innovation dans la Conception de formation), que consiste en un trabajo conjunto entre la Universidad de Sherbrooke (Canadá) y la Universidad de Bretagne Loire (Francia). Se trata de un estudio en línea (informatizado) realizado a 174 formadores de dos universidades asociadas que debían responder a la siguiente pregunta: ¿Cuál es la utilidad (o utilidades) actuales, el nivel de manejo y las expectativas de los formadores en relación con los dispositivos de tecnología pedagógica de tipo videoconferencia? El estudio reveló que los dispositivos de videoconferencia son poco utilizados y que los formadores no están preparados para utilizarlos. A pesar de todo, la mayoría de las personas que respondieron dicen sentirse capaces de enseñar en un contexto digital. En cuanto a la formación, los formadores privilegian los recursos que favorecen la autoformación en vez de un acompañamiento personalizado o una combinación de recursos de acompañamiento.

Palabras clave: telepresencia, videoconferencia, webconferencia, consejeros pedagógicos, dispositivos de tecnología pedagógica, formación continua, educación superior, desarrollo profesional

\section{Introduction}

De 2015 à 2019, l'Université de Sherbrooke au Canada (UdeS) et l'Université Bretagne Loire en France (UBL) ont travaillé conjointement au sein du projet TOPIC (Téléprésence comme OPportunité d'Innovation dans la Conception de formation). Ce partenariat réunissait chercheurs et conseillers/ingénieurs pédagogiques des deux institutions afin de mieux comprendre comment former à l'usage des dispositifs technopédagogiques de type visioconférence, soit tous les outils technologiques permettant de réunir un groupe de personnes dans un espace virtuel partagé et synchrone. Plusieurs laboratoires de recherche ont été mobilisés : le Centre de Recherche sur l'Education, les Apprentissages et la Didactique (CREAD) de l'Université Rennes 2, le Laboratoire d'Informatique de I'Université du Mans (LIUM) et PeDTICE de l'Université de Sherbrooke. Le projet TOPIC a été financé sur deux années, 2018-2019, par le Conseil Franco-Québécois de Coopération Universitaire, dans le cadre du programme Samuel-de-Champlain, et a également bénéficié du soutien financier du Conseil Régional de Bretagne et de l'Université de Bretagne Loire. Le présent article traite des résultats d'un questionnaire en ligne qui a été distribué aux formateurs des deux institutions d'enseignement au printemps 2017. Cette démarche avait comme objectif de dresser un portrait des usages actuels de ces dispositifs technopédagogiques (visioconférence, téléprésence et webconférence) dans ces institutions et des attentes des formateurs afin de faciliter leur usage.

Au lancement de TOPIC, l'UBL déployait le projet « UEB C@ampus » dont l'objectif était de construire un campus numérique multisite régional afin de mieux desservir les étudiants répartis sur le vaste territoire breton. Ce déploiement ciblait l'intégration de nouveaux équipements technopédagogiques. Nous parlons plus spécifiquement de : 
- Salles de téléprésence immersive : des salles de visioconférences immersives de 18 places avec deux grands écrans permettant de voir les personnes à distance à taille réelle; le son stéréo est particulièrement bien restitué, ce qui donne le sentiment d'immersion.

- Salles de téléprésence : des salles, d'une capacité maximum de 12,18 ou 40 personnes, équipées de trois écrans permettant de voir les personnes à distance à taille réelle.

- Salles de télé-enseignement : des salles d'une capacité de 30 à 40 personnes équipées d'un tableau de projection et d'un tableau blanc interactif. Une caméra est placée entre le tableau de projection et le tableau blanc interactif afin de filmer la salle et une deuxième caméra est placée sur le mur d'opposition pour filmer l'enseignant.

- Télé-amphithéâtres : des amphithéâtres, d'une capacité de 80 à 150 personnes, équipés de deux grands écrans permettant de voir les sites distants; deux caméras sont installées afin de capter l'enseignant sur la chaire ainsi que les étudiants dans la salle.

L'idée derrière le développement du campus numérique était de rendre ces équipements interconnectés et de s'appuyer sur une infrastructure de communication collaborative permettant aux usagers de réserver les équipements, d'enregistrer leurs sessions, de travailler en réseau et d'accéder à un service d'assistance. Or, l'usage grandissant des dispositifs technopédagogiques de type visioconférence en enseignement supérieur place de plus en plus de formateurs devant la nécessité de renouveler leurs pratiques d'enseignement. D'une part, ils doivent négocier des situations inédites comme celle de gérer simultanément des étudiants à distance et d'autres en face à face. D'autre part, malgré une utilisation grandissante de ces nouveaux dispositifs technopédagogiques, les formateurs, les conseillers/ingénieurs pédagogiques et les chercheurs ont besoin de clés de compréhension pour faire évoluer ces dispositifs d'enseignement (Lameul et Loisy, 2014). Ceux-ci ne sont pas toujours adaptés aux multiples besoins de l'enseignement et de l'apprentissage (Alhlak et al., 2012) et n'ont pas été conçus pour s'arrimer aux nombreuses méthodes et approches pédagogiques (Gillies, 2008; Lawson et al., 2010). Pour l'essentiel, il s'agit a priori de dispositifs technologiques destinés à la tenue de réunions.

À cette nécessité des formateurs d'adapter leurs pratiques d'enseignement à ces nouveaux dispositifs technopédagogiques s'ajoutent tous les autres défis auxquels font face les universités, dont la nécessité d'enseigner à de grands nombres d'étudiants extrêmement hétérogènes (De Ketele, 2010; Goastellec, 2014). À cela s'ajoute, lorsqu'il est question des enseignants-chercheurs, qu'il est commun de retrouver un corps professoral qui dispose d'une expertise disciplinaire reconnue sans pour autant posséder une formation en pédagogie (Bédard, 2006; Bélisle et al., 2016; Hativa, 2000; Lison et Jutras, 2014; Romainville, 2006). Qu'en est-il lorsqu'ils doivent en plus intégrer les technologies à leur enseignement?

De plus, les formateurs évoluent dans des établissements où les dispositifs technopédagogiques se renouvellent toujours plus rapidement, et ce, avec parfois peu de souci pour les formateurs et leur contexte d'enseignement, ce qui témoigne d'une prétention que les compétences nécessaires à leur usage viendront d'elles-mêmes (Albero, 2011). Dès le début du projet, l'équipe de TOPIC était particulièrement sensible à ce dernier point et partageait le constat que les offres actuelles de formation des formateurs en enseignement supérieur ne répondaient pas aux besoins émergents (Lameul et Loisy, 2014). Dès lors, il s'agissait de saisir l'opportunité que représentait le développement du campus numérique à l'UBL pour penser autrement l'offre de formation continue des établissements partenaires. Parallèlement, il s'agissait d'intégrer dans notre réflexion les conseillers/ingénieurs pédagogiques et de définir leur rôle dans le développement de ces dispositifs de formation continue. Par exemple, au lancement de TOPIC, au niveau de l'Université de Sherbrooke, le soutien pédagogique offert par les conseillers pédagogiques concernait principalement une offre d'ateliers de formation développés par le Service de soutien à la formation et des accompagnements individualisés. 
La prochaine section présente un cadre de référence afin d'introduire la notion de téléprésence en ce qui a trait aux salles de visioconférences immersives, ainsi qu'une typologie des dispositifs de formation, en plus de préciser ce que nous entendons par compétence numérique. Ensuite, nous introduisons la méthodologie ayant mené aux résultats de notre enquête. Finalement, nous proposons une nouvelle lecture de ces résultats lors de la discussion.

\section{Cadre de référence}

\subsection{Téléprésence}

En termes de téléprésence, le projet TOPIC s'intéresse plus particulièrement à l'utilisation de salles immersives de visioconférence. Dans ce contexte, la téléprésence peut être définie comme un espace d'interactions sociales médiatisées grâce aux technologies; il ne s'agit pas de la réalité virtuelle comme l'évoque Jézégou (2010), mais d'une utilisation optimale du son et de la vidéo afin de créer cette fameuse présence à distance : " [...] ces technologies offrent la possibilité d'expérimenter une communication collective proche de la celle du présentiel notamment lors d'activités collaboratives à distance [...] » (Jézégou, 2010, p. 258). Ainsi, dans une salle de téléprésence, les apprenants devraient voir le formateur (et leurs pairs) à l'écran de la bonne grandeur, et ils devraient les entendre comme il faut, comme s'ils étaient dans la pièce avec eux. En ce qui a trait aux dispositifs de visioconférence réguliers, ils concernent également les salles équipées de tels dispositifs : caméras et téléviseurs. Par contre, ces salles ne disposent pas des qualités immersives propres à celles de téléprésence.

Parmi les espaces technologiques de type visioconférence, on peut distinguer trois grands ensembles de dispositifs technologiques:

- Les dispositifs de téléprésence, qui font référence à des salles immersives permettant un rapport $1 / 1$ et disposant d'une qualité sonore supérieure.

- Les dispositifs de visioconférence réguliers qui se rapportent à des salles de classe adaptées et munies de caméras et de téléviseurs (sans de rapport 1/1).

- Les dispositifs de webconférence qui concernent les dispositifs de type classe virtuelle où les personnes participent en ligne depuis un ordinateur personnel.

Les dispositifs de webconférence sont des environnements numériques et virtuelles qui permettent une communication en temps réel (Macedo-Rouet, 2009) entre un formateur et une classe, et même entre les étudiants, et ce, tout en mettant à la disposition des utilisateurs diverses fonctions de collaboration (Poellhuber et al., 2012). Ces environnements reposent sur des logiciels, tel qu'Adobe Connect, Via ou Elluminate et sont particulièrement propices à véhiculer un sentiment de présence transactionnelle (Poellhuber et al., 2012). C'est-à-dire qu'ils concernent le degré auquel des étudiants perçoivent la disponibilité des formateurs, de leurs pairs ou de l'établissement et le degré auquel il se perçoit en lien avec eux (Shin, 2002). Ainsi, dans la mesure où les dispositifs de téléprésence se distinguent par la qualité de l'expérience qu'ils offrent aux étudiants et aux formateurs, les dispositifs de webconférence concernent davantage l'aspect transactionnel de la présence à distance. 


\subsection{Dispositif de formation continue}

Afin de soutenir les formateurs dans l'adaptation de leur enseignement aux dispositifs technopédagogiques de type visioconférence, plusieurs types de dispositifs de formation peuvent être mis en place. Dans le cadre de cet article, nous allons nous appuyer sur la typologie proposée par Albero (2014) dont la catégorisation a été produite à partir de plusieurs études portant sur une variété de dispositifs de formation (Albero, 2000) intégrant ou non le numérique. Bien que cette typologie ait été développée pour comprendre les pratiques d'enseignement en général, nous l'appliquons dans cet article à un contexte de formation continue. Pour éviter les confusions, nous parlons donc de dispositif de formation continue.

Cette typologie distingue quatre idéaux-types; le prescriptif, le tutoral, le coopératif et l'autodirectif (figure 1). Chacun de ces idéaux-types disposent de "caractéristiques spécifiques: contenus de formation, types d'intervenants et fonctions, modes d'évaluation, traitement de l'espace et du temps, fonction (des objets techniques) » (p. 45). Ils se situent sur un continuum entre des pratiques sociales marquées, d'un côté, par l'instruction et, de l'autre, par l'autonomie.

Ces quatre catégories de dispositif de formation se rapportent à trois approches pédagogiques. Premièrement, la pédagogie de la transmission centrée sur la transmission de contenus et sur la mise en ligne de ressources. Deuxièmement, la pédagogie de l'entraînement, qui vise la transmission d'une « manière de faire » en offrant un soutien aux formateurs et une scénarisation des ressources. Cette approche est centrée sur l'intervention. Finalement, une pédagogie du développement qui soutient les apprentissages et l'autoformation. Nous parlons d'une pédagogie centrée sur les apprentissages.

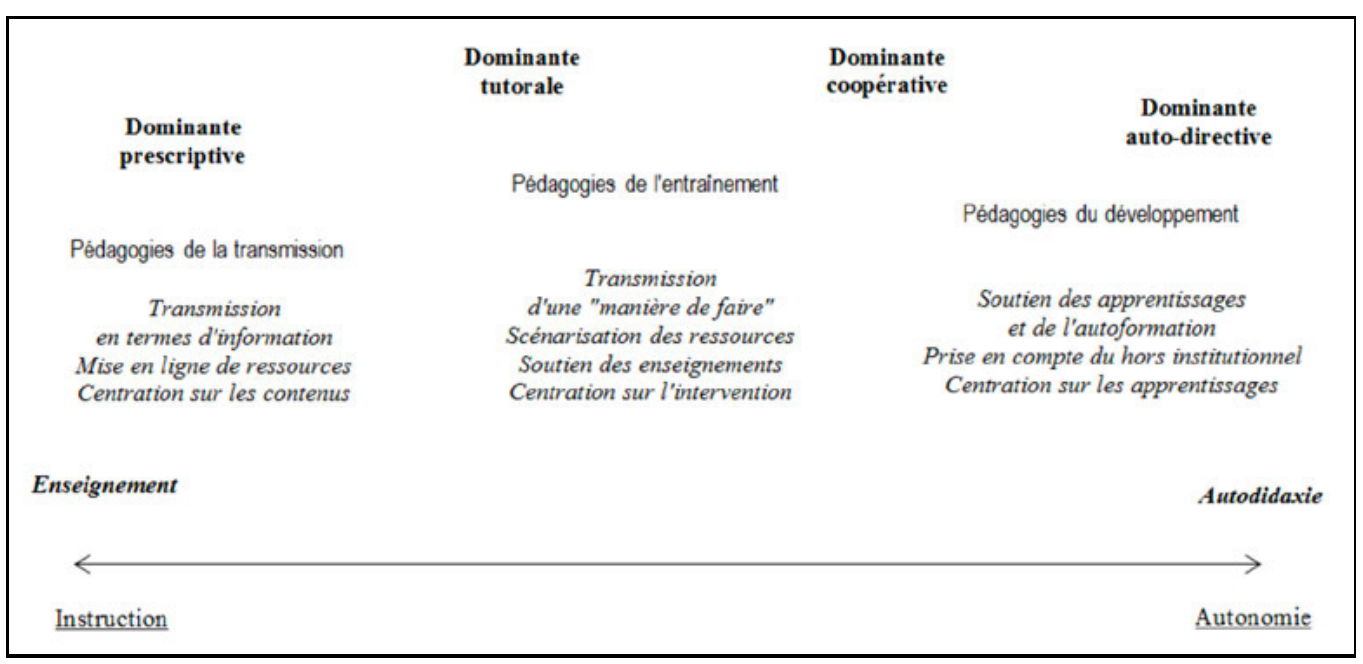

Figure 1

Typologie des dispositifs de formation selon les paradigmes d'inscription et leurs visées tiré de Albero (2014)

Dans le cadre de cet article, cette typologie nous permet de mieux situer les attentes des formateurs universitaires en termes de la formation qu'ils souhaitent obtenir quant à l'utilisation des dispositifs technopédagogique de type visioconférence. 


\subsection{Compétences numériques et intégration des TIC}

L'appropriation des dispositifs technopédagogique de type visioconférence par les formateurs dépend également de leurs compétences numériques. Afin de mettre en œuvre une utilisation efficace et riche des technologies dans sa pratique, un formateur doit faire appel à de multiples connaissances lui permettant d'agir avec compétence et de faire des choix appropriés dans diverses situations de pratique. Afin de distinguer ces différentes connaissances, Mishra et Koehler (2006), s'appuyant sur le modèle Pedagogical Content Knowledge (PCK) développé par Shulman (1986, 1987), proposent le Technological Pedagogica and Content Knowledge (TPACK). II s'agit d'un modèle, comme la présente la figure 2, distinguant trois grandes familles de connaissances : les connaissances technologiques (Technological Knowledge), les connaissances pédagogiques (Pedagogical Knowledge) et les connaissances de contenu (Content Knowledge) dont Bachy (2014) confirme la complexité et l'hétérogénéité en enseignement supérieur compte tenu des multiples disciplines et de leurs ancrages et cultures très variées. Bien entendu, selon les contextes et les situations de pratiques, ces familles de connaissance sont plus ou moins mobilisées par les formateurs.

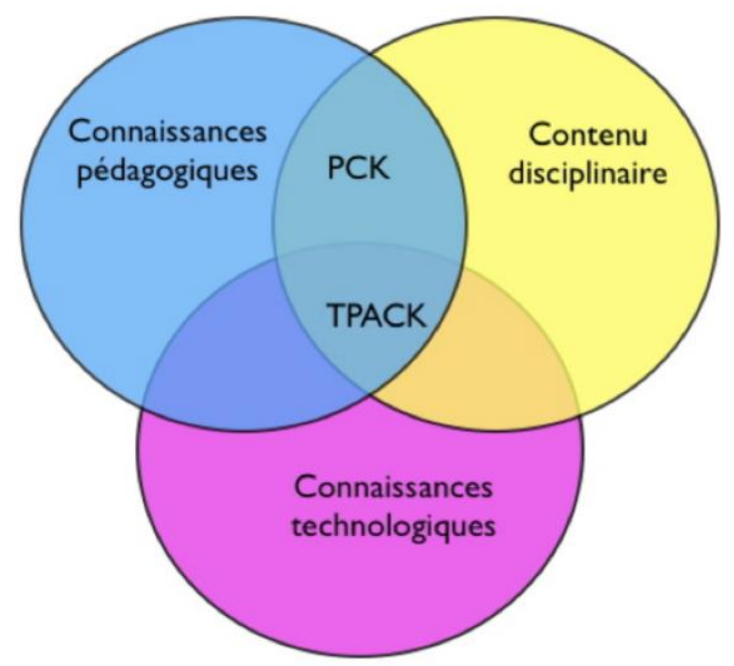

Figure 2

Modèle TPACK adapté de Mishra et Koehler (2006) et tiré de Bachy (2014).

Lorsque nous évoquons l'idée de mobilisation des connaissances dans des contextes technopédagogiques, il est indispensable de comprendre le contexte dans lequel les enseignants doivent intervenir (Mishra et Koehler, 2006). À cet égard, Villeneuve (2011) a développé un modèle de facilitateurs à l'intégration des technologies de l'information et de la communication (TIC). Ce modèle (voir figure 3), bien qu'il concerne avant tout les enseignants du primaire et du secondaire, distingue les facilitateurs provenant de dimensions plus personnelles (Personnel) et les facilitateurs provenant de ce qu'il appelle les dimensions politiques (Politique), c'est-à-dire les facilitateurs externes à l'enseignant. Dans sa recherche, Villeneuve (2011) a démontré que les facilitateurs politiques comprennent la formation et les informations reçues par un enseignant, les décisions administratives de l'établissement dans lequel cet enseignant exerce, la gestion de l'établissement et des infrastructures technologiques notamment et l'accès aux ressources humaines ou matérielles. 


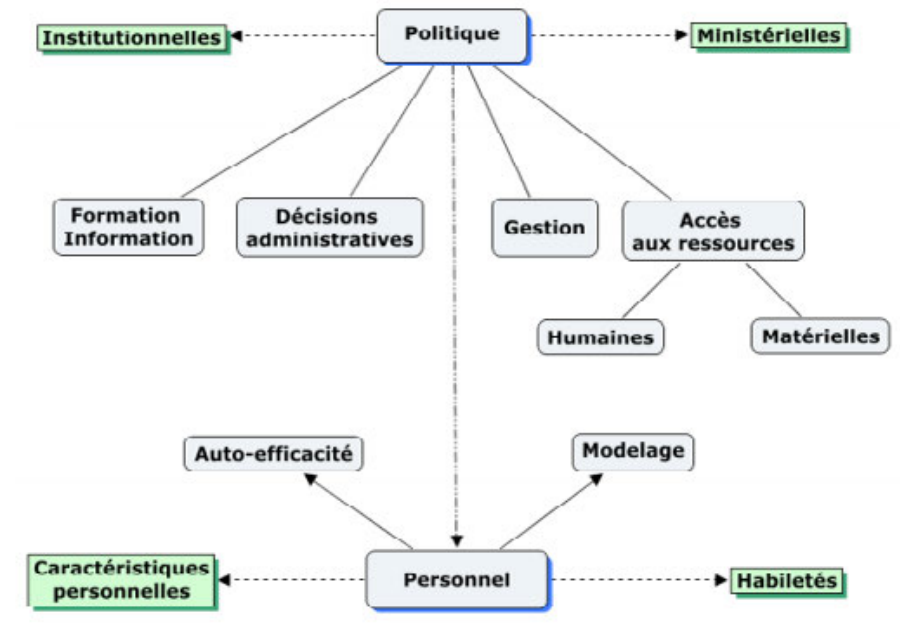

Figure 3

Facilitateurs à l'intégration des TIC (Villeneuve, 2011, p. 178)

Ainsi, les dimensions politiques ont une répercussion forte sur les dimensions personnelles. Ces dernières, Villeneuve les distingue en quatre grands ensembles : les caractéristiques personnelles propres à chaque enseignant, son sentiment d'auto-efficacité à l'égard de l'intégration des TIC, sa capacité à se sentir mobilisé par les exemples et modèles observés et ses habiletés et connaissances technologiques.

\section{Méthodologie}

Au printemps 2017, une des premières actions de l'équipe TOPIC a été de mener une enquête en ligne auprès des formateurs membres des deux établissements partenaires : I'UdeS et l'UBL. Cette enquête avait pour objectifs de : 1) de mieux connaître les usages des dispositifs technopédagogiques de type visioconférence; 2) de cerner le niveau d'aisance des formateurs avec les technologies, leur niveau de formation actuel, et 3 ) de préciser leurs attentes en termes de formation, d'information et de ressources pour l'usage de ces technologies. De manière plus spécifique, elle visait à répondre à la question de recherche suivante : "Quels sont les usages actuels, le niveau d'aisance et les attentes des formateurs face aux dispositifs technopédagogiques de type visioconférence? ».

L'enquête en ligne a démarré par une invitation qui a été envoyée par les services de soutien pédagogique des deux universités partenaires à l'ensemble de leurs formateurs (professeurs et chargés de cours). Au final, des 205 répondants, seuls ceux catégorisés "enseignant-chercheur » ou " professeur » ont été retenus, soit un total de 174 répondants. L'objectif était de retenir que ceux qui disposaient de tâches d'enseignement. De ce nombre, une forte majorité, soit 121 répondants, provenait de l'UBL. De plus, comme la présente la figure 4 , au total, $55 \%$ des participants enseignaient dans une discipline des sciences humaines et sociales (SHS), $38 \%$ des sciences naturelles et du génie (SNG) et $7 \%$ des sciences de la santé (SS). 


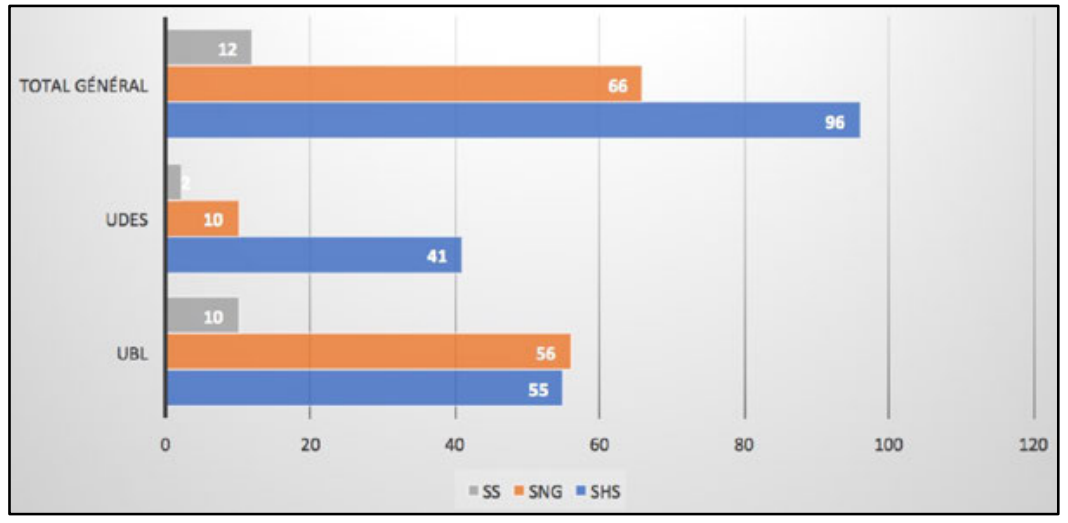

Figure 4

Discipline d'origine des répondants en fonction des établissements

L'enquête s'inscrit dans une démarche de recherche de nature descriptive et exploratoire avec une approche méthodologie mixte combinant l'analyse de données quantitatives et qualitatives. Le questionnaire utilisé comporte vingt questions à choix de réponses et trois questions à court développement. L'échantillon est de nature circonstancielle, de convenance (Pires, 1997) et repose sur un échantillonnage non probabiliste. Le faible échantillon ne nous permet pas de généraliser ces résultats aux contextes des autres institutions d'enseignement supérieur tant en France qu'au Canada.

Le volet quantitatif vise à préciser le profil des participants, à déterminer leur fréquence d'utilisations des trois dispositifs technopédagogiques, leur niveau de formation, leur aisance avec les technologies et leur usage de technologies complémentaires. La fréquence d'utilisations a été analysée à l'aide d'une échelle de Likert à 5 niveaux : "Au moins une fois par an », "Au moins une fois par semestre », " Au moins une fois par mois », "Au moins une fois par semaine » et « Jamais ».

Une analyse qualitative a été effectuée sur les trois questions à court développement, qui concernaient les attentes des répondants en termes de formation, de ressources, d'informations et de technologies ou d'outils complémentaires. Pour ce faire, nous avons réalisé une analyse thématique (Paillé et Mucchielli, 2016) et dressé un panorama de la diversité des attentes des répondants. Pour chacune des trois questions à court développement, une première étape d'analyse a permis de regrouper les réponses sous des thèmes précisant l'objet des propos des participants. Nous avons donc cherché à faire ressortir dans cette première étape à quoi la personne faisait référence. Par exemple, sur la question des attentes en termes de formation, trois thèmes mutuellement exclusifs ont été déterminés, soit le type de formation souhaitée, les caractéristiques de ces formations et les objets de ces dernières. Ainsi, de cette première étape, il ressort que les attentes en termes de formation de certains répondants concernent davantage l'approche pédagogique qui sera utilisée pour ces formations (type de formation), tandis que d'autres pointent des qualités souhaitées pour ces formations (caractéristiques des formations), comme un « temps de formation réduit ", de la " souplesse et facilité d'accès » ou " des formations courtes ciblées sur des points précis ». Par la suite, une deuxième étape d'analyse a permis d'identifier des sous-thèmes pour chacun de ces thèmes. Dès lors, nous avons procédé par saturation où un nouveau thème était généré à chaque fois que les propos des répondants spécifiaient un élément distinct. L'objectif était de générer des sous-thèmes mutuellement exclusifs. Par exemple, à la question sur les attentes en termes de formation, l'analyse des réponses regroupées sous le thème "type de formation ", nous a permis de générer le thème " autoformation » et regroupé des réponses comme " Des démonstrations par des vidéos ou des tutoriels faciles d'accès sur internet », "Tutoriels » et " Être mis au courant des nouvelles fonctionnalités avec, au besoin, des tutoriels en ligne ». 
Cette analyse thématique nous a permis de dresser un portrait tant des usages que des attentes des formateurs en nous informant toutefois peu sur le contexte d'usage. Par exemple, au-delà de pouvoir spécifier qu'un tel dispositif est utilisé dans le cadre d'un cours, les résultats ne nous permettaient pas d'en préciser davantage. De plus, une des limites de notre analyse est d'avoir été appliquée sur un corpus où la grande majorité des réponses consistaient en quelques mots. Sommes toutes, les résultats proposent un portrait pertinent au regard d'une démarche exploratoire.

\section{Résultats}

Les données colligées auprès de 174 formateurs des deux universités partenaires ont permis de préciser le profil des participants et leurs usages des dispositifs technologiques de type visioconférence en contexte de formation à distance. Également, de cerner leurs attentes en termes de formation, de ressources, d'informations, de technologies et d'outils complémentaires.

\subsection{Niveau d'expérience, aisance et formation}

La grande majorité des participants, soit $80 \%$ d'entre eux, disposent de plus de 10 années d'expérience en enseignement. Or, seulement $15 \%$ affirment avoir été formé à l'usage des dispositifs technologiques et, de ces personnes, $85 \%$ ont participé à une ou deux formations. Également, les participants se disent relativement à l'aise avec le numérique en général, soit $71 \%$ se considèrent " À l'aise " ou "Très à l'aise ». Or, ce pourcentage passe à $61 \%$ lorsqu'il est question de leur niveau d'aisance face à leur capacité à enseigner avec le numérique. Cette différence de $10 \%$ concerne particulièrement le groupe des personnes qui se disent «Peu à l'aise » dont le nombre augmente lorsqu'il est question de la capacité des personnes à enseigner avec le numérique. Or, au final, malgré le peu de formation reçue pour l'utilisation des dispositifs étudiés, une forte majorité des répondants $(61 \%)$ se disent à l'aise à l'utilisation des nouvelles technologies pour enseigner.

\subsection{Fréquence et cadre d'usage}

Pour les répondants, la téléprésence demeure un dispositif peu utilisé : $3 \%$ des répondants l'utilisent une fois par semaine et $71 \%$ ne l'utilisent jamais. Notons que seule l'UBL comptait de ce genre de salles. Or, comme le présente la figure 5 , même les dispositifs les plus utilisés, soit la visioconférence ( $11 \%$ l'utilisent une fois par semaine) et la conférence web (17\% l'utilisent une fois par semaine), le sont avant tout pour des réunions de travail. Cette tendance est d'autant plus marquée à l'UBL où l'utilisation pour des rencontres individuelles et pour des évaluations dépasse leur usage dans le cadre de cours.

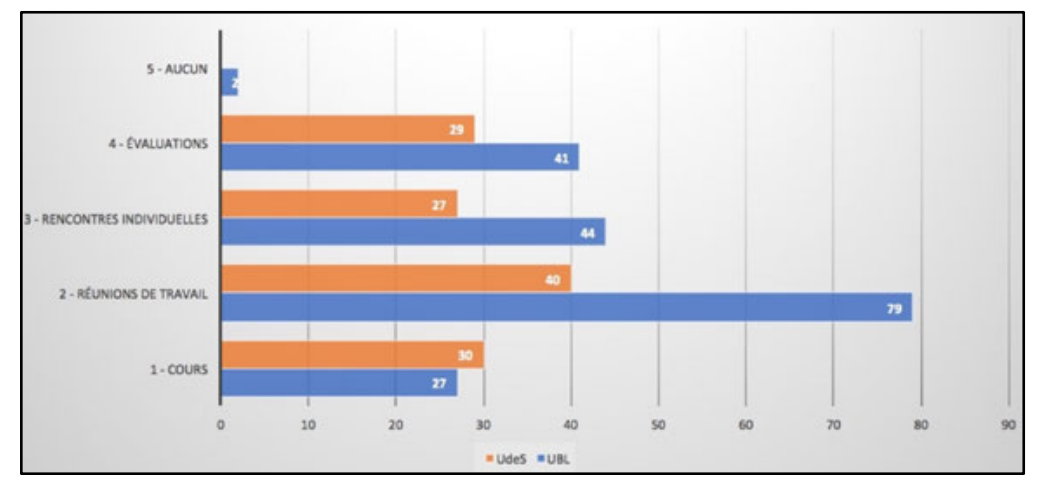

Figure 5

Cadre d'utilisation des dispositifs de type visioconférence 


\subsection{Attentes en termes de formation}

Cinq catégories ont émergé de l'analyse des réponses à court développement sur les attentes en termes de formation (111 répondants sur les 174 participants). Comme le dévoile la figure 6 , une première catégorie regroupe les répondants qui traitent des objets des formations à privilégier $(35 \%)$; une deuxième, les personnes qui n'avaient aucune attente (27\%); une troisième, ceux qui se réfèrent aux types de formations à envisager ( $24 \%$ ); une quatrième, qui cible les caractéristiques des formations souhaitées $(8 \%)$ et, finalement, une cinquième catégorie dont les répondants confirmaient uniquement leur intérêt envers une formation (6\%).

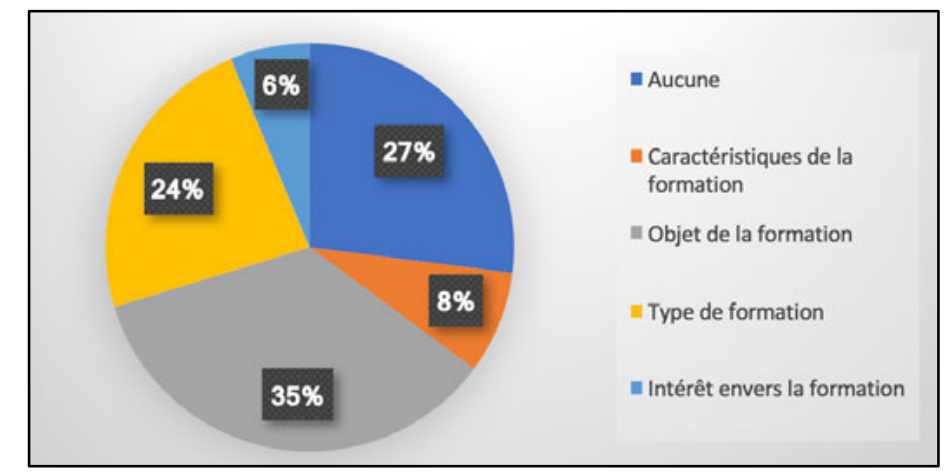

Figure 6

Les attentes des répondants concernant les formations offertes au sein de leur institution

Plus spécifiquement, pour ce qui est des attentes face au type de formation, comme la présente la figure 7 , des 26 répondants regroupés dans cette catégorie, $34 \%$ préfèrent l'autoformation, $27 \%$ l'accessibilité à un accompagnement au besoin, $15 \%$ aimeraient pouvoir obtenir une formation de base qui serait jumelée à un accompagnement, $8 \%$ souhaiteraient que les formations proposent un travail pratique et concret et $8 \%$ opteraient pour une formation avec un volet individuel et un autre collectif. Finalement, une seule personne désire une formation intensive et une autre une formation de type magistral.

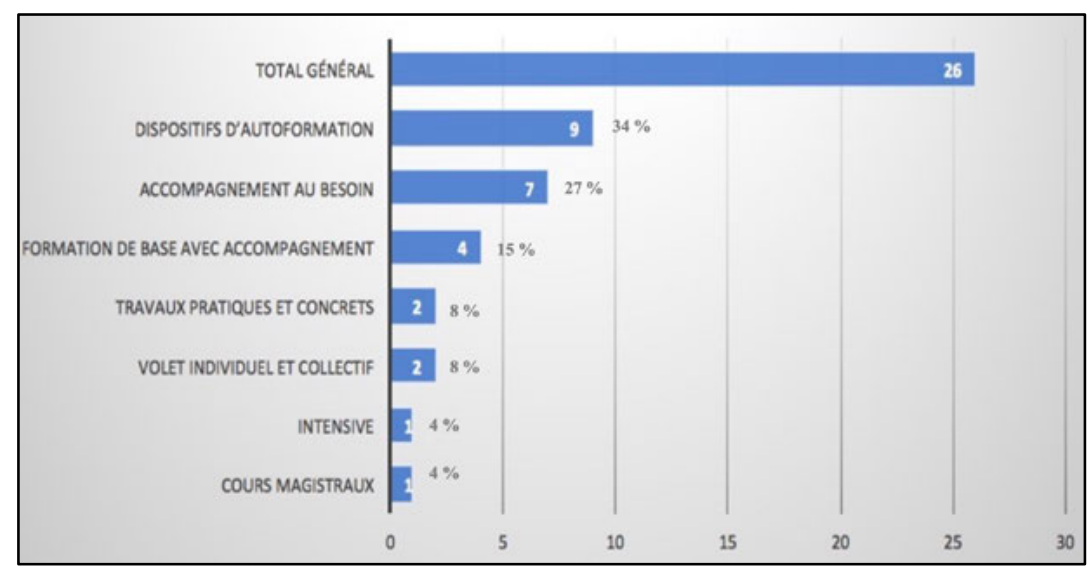

Figure 7

Les attentes des répondants concernant les types de formation 
Ensuite, comme l'introduit la figure 8, lorsque le besoin de formation est identifié, des 39 répondants regroupés dans cette catégorie, $46 \%$ privilégient des formations dont l'objet concerne l'utilisation des outils et des fonctionnalités, $33 \%$ disposaient d'un intérêt pour des formations sur l'enseignement et l'apprentissage, $13 \%$ optaient pour une combinaison de formation sur les aspects techniques et pédagogiques, $5 \%$ désiraient une formation continue et suivre l'évolution des technologies et $3 \%$ souhaitaient que les formations portent sur des retours d'expérience de leurs collègues.

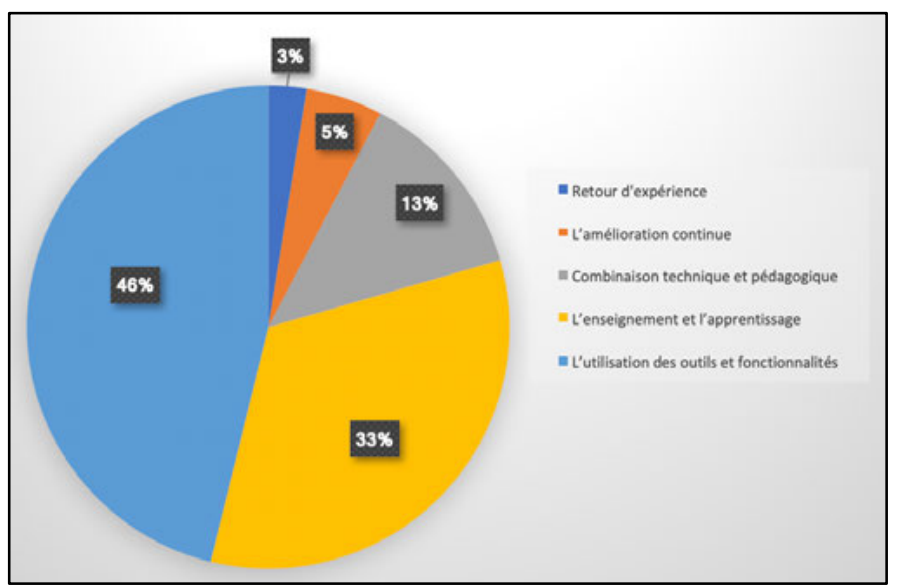

Figure 8

Les attentes des répondants concernant les objets de formation

Finalement, des 9 répondants qui ont traité des caractéristiques des formations, $88 \%$ privilégient des formations en format réduit, qui sont efficaces et facilement accessibles. Une seule personne privilégie pour sa part des formations qui partent des besoins des formateurs. 


\subsection{Attentes en termes de ressources et d'information}

Six catégories ressortent de l'analyse qualitative des réponses à court développement sur les attentes en termes de ressources et d'informations (93 répondants sur les 174 participants). Comme la présente la figure 9 , des 93 répondants, $53 \%$ font référence aux types de ressources et d'informations qu'ils désiraient, $25 \%$ n'avaient aucune attente, $9 \%$ traitaient des qualités désirées des ressources et des informations, $7 \%$ des types d'équipements à rendre accessibles, $5 \%$ proposaient des thèmes ou des objets de formation et $1 \%$ affirmait avoir des attentes fortes.

Par exemple, dans les propositions de thèmes ou d'objets de formations, nous retrouvons des formations sur les réseaux sociaux, la mise en forme de vidéo ou, sous forme de thématique, l'exploration des possibilités des ressources disponibles. À l'égard des types d'équipement, une diversité d'équipements est suggérée, dont des salles immersives (soit de téléprésence), des salles de visioconférence ou l'accès à Internet. Pour ce qui est des qualités désirées, une liste faisant état de qualités variées ressort, dont principalement la facilité, la fiabilité, la simplicité d'usage et l'autonomie sur le système.

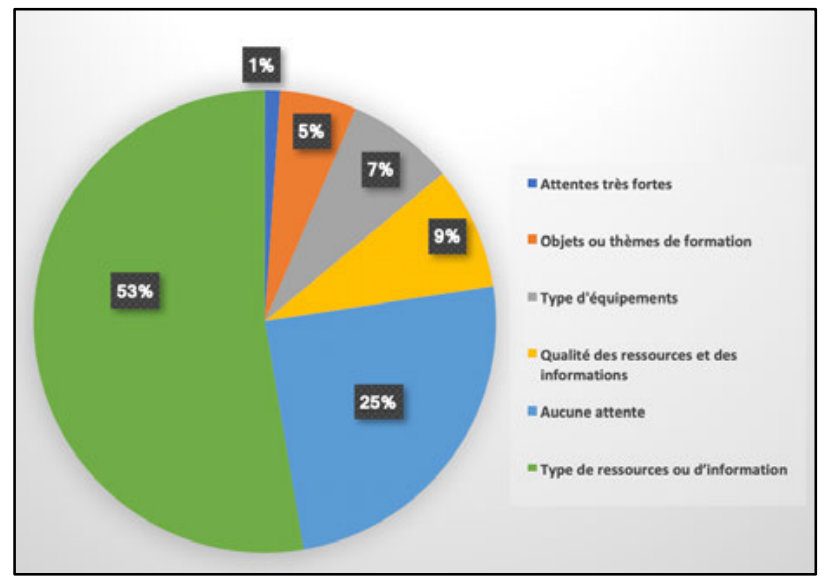

Figure 9

Attentes des participants en termes de ressources et d'information

En termes de ressources et d'informations, comme le présente la figure 10, des 49 répondants, $33 \%$ privilégient spécifiquement un accès facile et direct à un soutien technique, $25 \%$ proposent la mise à leur disposition d'une combinaison de ressources, d'information et d'accompagnement personnalisé, $9 \%$ mentionnent l'accès à des tutoriels, des guides ou d'autres dispositifs d'autoformation, $9 \%$ à un accès à de l'information et, finalement, $6 \%$ font référence à des formations et ces derniers insistent sur le caractère court et pratico-pratique de ces dernières. Dans les combinaisons proposées, une forte majorité, soit 10 des 12 répondants, réfère à la présence d'un technicien ou d'un accompagnement avec d'autres ressources, dont des tutoriels, des guides ou documents explicatifs. 


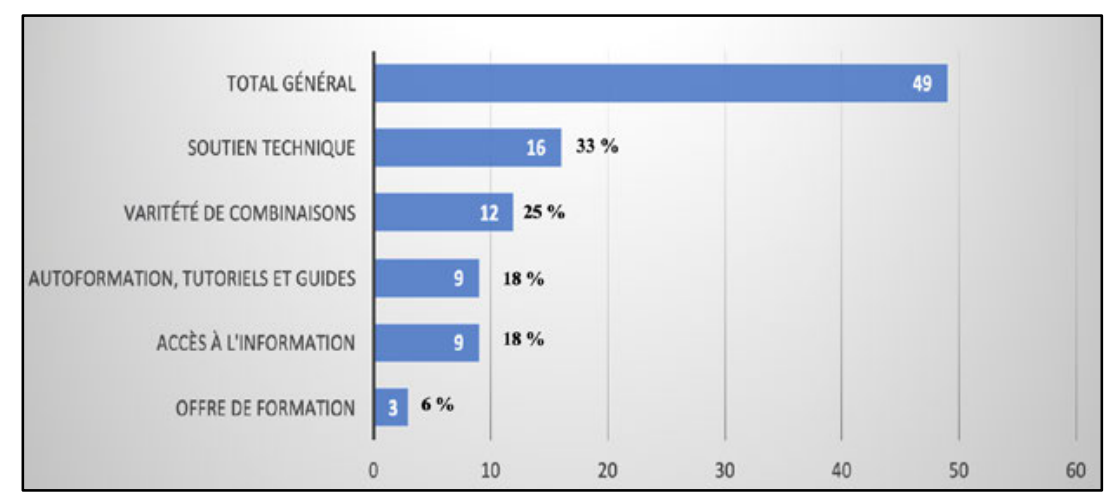

Figure 10

Répartition des types de ressources et d'information

\subsection{Attentes en termes de technologies ou d'outils complémentaires}

Six catégories ressortent de l'analyse qualitative des réponses à court développement sur les attentes en termes de ressources et d'informations (87 répondants sur les 174 participants). Comme le présente la figure 11, des 87 répondants, $45 \%$ énuméraient diverses qualités souhaitées, $31 \%$ n'avaient aucune attente, $12 \%$ proposaient des équipes spécifiques, $8 \%$ désiraient un accès à des ressources ou de l'information, $3 \%$ traitaient d'objectifs d'apprentissage ou type d'utilisation souhaitée et $1 \%$ avait des attentes très fortes.

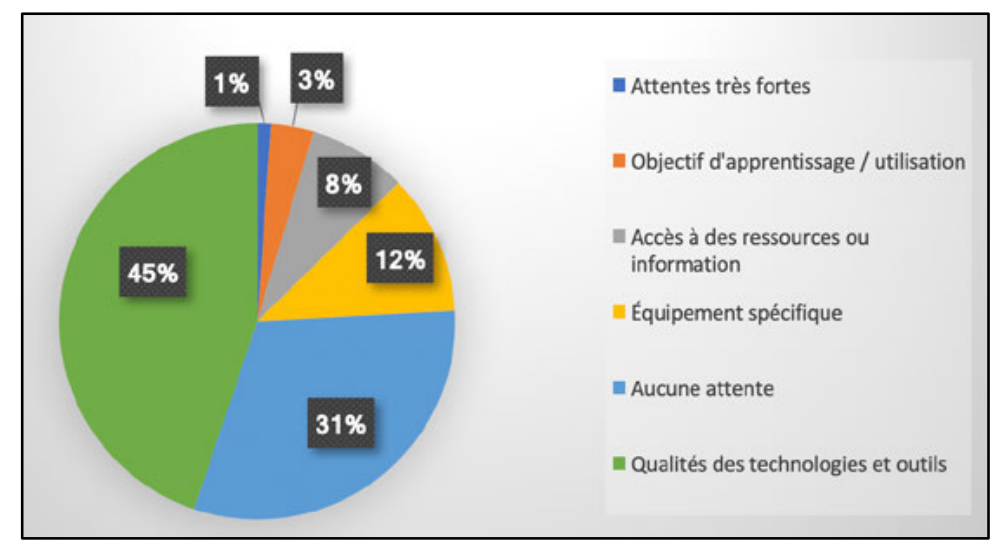

Figure 11

Attentes en termes de technologies et d'outils complémentaires

La figure 12 présente les résultats détaillés des qualités des technologies et des outils souhaités. Les qualités les plus demandées sont l'accessibilité ou la disponibilité $(28 \%)$, la fiabilité $(28 \%)$ et la compatibilité $(13 \%)$. Ensuite, les qualités indiquées sont la simplicité $(10 \%)$, l'interactivité et la collaboration ( $8 \%$ ), la flexibilité et le caractère modulaire (5\%), une meilleure qualité $(5 \%)$ et, finalement, des technologies et des outils qui complètent l'approche pédagogique des formateurs (3\%). 


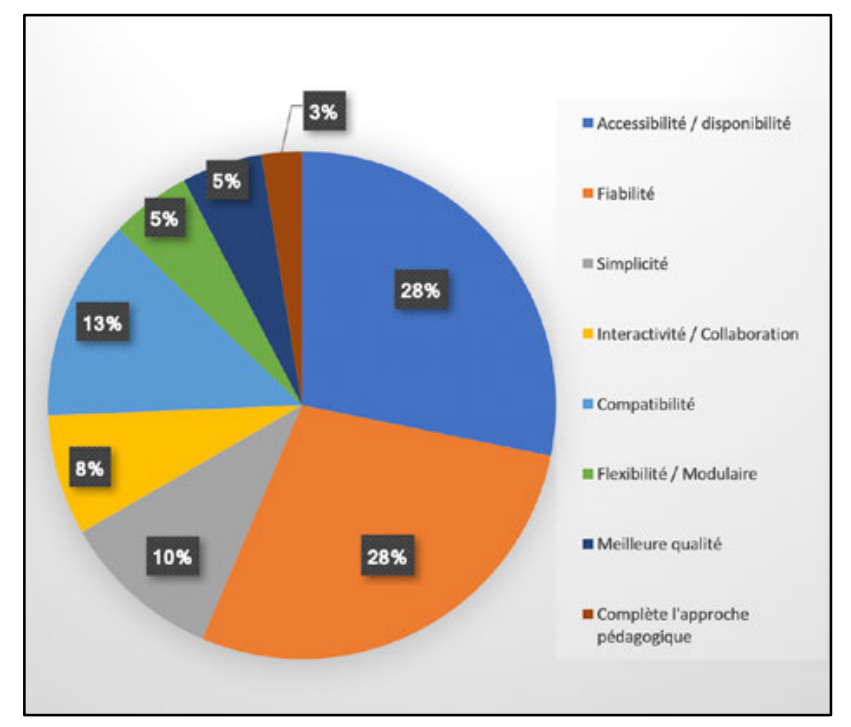

Figure 12

Qualités souhaitées des technologies et outils complémentaires

\subsection{Synthèse des résultats}

En synthèse, il ressort de nos résultats que les formateurs utilisent peu les dispositifs technopédagogiques qui sont à leur disposition. Par exemple, en ce qui a trait aux deux dispositifs les plus utilisés, la visioconférence l'est une fois par semaine par $11 \%$ des répondants et la conférence web, par $17 \%$ de ces mêmes répondants. Également, 71 \% des répondants affirment ne jamais utiliser les dispositifs de téléprésence. Cependant, comme le présente la figure 5 , lorsque ces dispositifs sont utilisés, ils le sont avant tout pour des réunions de travail.

En termes de formation, bien que seulement $15 \%$ des répondants aient reçu une ou deux formations pour enseigner avec un de ces dispositifs technologiques, $71 \%$ se considèrent " À l'aise " ou "Très à l'aise » à intégrer le numérique dans leur enseignement. Aussi, lorsqu'ils sont interrogés sur leurs attentes, $27 \%$ des répondants déclarent n'avoir aucune attente en termes de formation et lorsqu'ils énoncent des attentes, elles concernent surtout des objets de formations (35\%) ou précisent le type de formation souhaitée $(24 \%)$. Dans le dernier cas, comme le présente la figure 7 , les formateurs souhaitent majoritairement accéder à des dispositifs d'auto-formation (34 \%) ou à un accompagnement au besoin (27\%).

En ce qui concerne les ressources et les informations, les attentes de $53 \%$ des répondants font référence au type de ressources ou d'informations souhaité. II est alors question d'accéder à un soutien technique (33\%), de proposer diverses combinaisons de soutien technique et de dispositifs d'auto-formation (25\%) ou d'accéder à des guides, tutoriels ou autres dispositifs d'auto-formation (9\%). Seulement $6 \%$ font référence à des formations.

Finalement, pour ce qui est des attentes envers les technologies et les outils complémentaires, hormis que $31 \%$ des répondants n'avaient aucune attente, $45 \%$ des répondants mentionnent certaines qualités recherchées de ces technologies et outils. Principalement, ils font référence à l'accessibilité ou la disponibilité ( $28 \%$ ), la fiabilité ( $28 \%$ ) et la compatibilité (13\%) de ceux-ci. 


\section{Discussion}

À la lumière de l'analyse de nos résultats de recherche, nous dégageons trois constats à l'égard des besoins de formation continue des formateurs des deux institutions partenaires et de leur utilisation des dispositifs de téléprésence, visioconférence et webconférence.

\subsection{Téléprésence : un phénomène encore marginal}

Malgré la présence grandissante du numérique dans nos établissements d'enseignement, il ressort de l'enquête réalisée que l'usage des dispositifs de type visioconférence en salle de classe demeure encore marginal. C'est tout particulièrement le cas pour les salles de téléprésence. Ainsi, bien qu'il existe de tels dispositifs dans les deux établissements étudiés, il apparaît que leur usage aux fins d'enseignement n'est que peu répandu chez les formateurs.

Pourtant, la formation à distance s'impose de plus en plus en enseignement supérieur (Bates et al., 2019). Par exemple, au Canada, le sondage national de 2018 sur la formation à distance et l'apprentissage en ligne révèle « une hausse importante du nombre d'établissements offrant des cours en ligne entre 2010 et 2011 (de $68 \%$ à $76 \%$ ), puis une augmentation plus graduelle entre 2011 et 2016 (de $76 \%$ à $79 \%$ ) » (Bates et al., 2019, p. 7). D'ailleurs, toujours selon Bates et al. (2019), « la majorité des établissements estiment que l'apprentissage en ligne est essentiel à leur avenir, et veillent à ce que ce type d'apprentissage soit de haute qualité » (p. 8). Ces constats nous confirment l'intérêt de poursuivre les recherches sur l'usage et les attentes envers ces dispositifs et d'approfondir notre compréhension des particularités de ces contextes de formations.

\subsection{Une intégration des ressources dans les environnements d'apprentissage et d'enseignement}

Notre deuxième constat est que les formateurs veulent retrouver dans leur environnement d'enseignement une réponse à leurs besoins en termes de formation, d'information ou de ressources. Ils sont peu intéressés à des formations sur différents sujets et veulent être soutenus le plus possible au niveau de leur propre activité d'enseignement. Si nous reprenons la typologie d'Albero (2014), les attentes des participants correspondent donc moins à des dispositifs de formation à dominante prescriptive, caractérisés par une pédagogie de transmission et se rapportent davantage à des dispositifs de formation à dominante auto-directrice centrés sur une pédagogie du développement.

Nous retrouvons ce même constat dans d'autres études portant sur l'intégration des technologies en enseignement (Glover et al. 2016; Kukulska-Hulme, 2012). D'ailleurs, la littérature dans ce domaine fait état d'une transition vers des approches centrées sur le développement continu des formateurs (Murthy et al., 2015; Viberg et al., 2018) où l'objectif est moins de tenter de répondre à un besoin ponctuel des formateurs (quick learning) que d'implanter des réponses institutionnelles dans le but de soutenir les formateurs dans leur développement professionnel.

À ce titre, O'Donnell et al. (2012) insistent sur trois principes pour orienter le développement de ressources destinées à soutenir les formateurs : 1) se référer à des exemples qui sont ciblés localement; 2) adopter une approche consultative de développement; 3 ) développer des ressources non prescriptives. Pour leur part, Glover et al., (2016) suggèrent que toute démarche de conception de ressources technopédagogiques doit tenir compte et s'appuyer sur les pratiques existantes des formateurs. À titre d'exemple, Glover et al., (2016) décrivent une démarche en trois étapes pour soutenir le développement 
des pratiques technopédagogique de formateurs universitaires. Dans un premier temps, ils ont réalisé un sondage anonyme pour recueillir les pratiques technopédagogiques actuelles des formateurs. Les résultats de ce sondage ont permis d'identifier les approches pédagogiques et les technologies utilisées et de créer un premier cadre de référence. Dans un deuxième temps, un atelier de travail a été proposé afin que les formateurs puissent discuter et adapter ce cadre de référence. Selon ces auteurs, cet atelier de travail a permis autant de construire un langage commun au sein de l'établissement que d'identifier des formateurs dont les pratiques pourraient servir à produire des études de cas destinées à imager certaines pratiques. Suite à cet atelier de travail, une première version d'un "menu » de ressources a été diffusée en ligne avec la possibilité de commenter et proposer des adaptations. Finalement, des ateliers de travail ont été organisés pour les groupes dont les formateurs désiraient changer leurs pratiques technopédagogiques.

Dans une perspective de développement continu des formateurs, si nous revenons à notre réflexion sur le rôle des conseillers/ingénieurs pédagogiques, il apparaît que ce rôle consiste moins à développer des ateliers de formation sur l'usage des dispositifs de téléprésence, visioconférence et webconférence, que de contribuer à la création de ressources fondées sur les pratiques actuelles et destinées à soutenir les formateurs dans leur activité d'enseignement.

\subsection{Rôle des formateurs face aux changements technopédagogiques des institutions}

Face aux résultats de la présente enquête où les participants semblent privilégier un soutien technique plutôt que technopédagogique, notre premier réflexe a été de penser que les formateurs ne voyaient pas forcément l'intégration de nouvelles technologiques dans leur enseignement comme une occasion de réfléchir sur leur pédagogie. Leurs attentes pointaient davantage vers l'amélioration de leurs connaissances technologiques et moins vers ce que Mishra et Koehler (2006) nomment le technological and pedagogical content knowledge (TPACK). Or, il est aussi possible que cette tendance observée soit une réponse des formateurs face au fonctionnement de leur établissement d'enseignement qui implante de nouvelles technologies sans réellement prendre en compte leurs contextes d'enseignement (Glover et al., 2016). Cette tendance, décrite par Albero (2011) et d'autres chercheurs cités par cette dernière (Albero, 2004; Baron et Bruillard, 1996; Cuban, 1986; Jacquinot, 1985; Jacquinot et Fichez, 2008; Linard,1996; Thibault, 2007; Wallet, 2007), relève d'expérimentations technopédagogiques qui se succèdent dans les institutions d'enseignement supérieur sans réel approfondissement et prise en compte des questions pédagogiques. Selon Albero (2011), ces démarches d'expérimentation placent les formateurs comme des « acteurs passifs de processus éphémères [...] privés de leur capacité d'action, d'initiative et de réflexion critique » (p. 15).

À l'instar des travaux d'Albero (2011) et du modèle de Villeneuve (2011), nos résultats nous portent à croire que les facilitateurs " politiques " qui orientent l'implantation de projets technopédagogiques institutionnels, comme celui du campus numérique de l'UBL, ont de fortes répercussions sur les facilitateurs " personnels " de ce processus. Pour l'équipe de TOPIC, ceci pose la question du rôle que nous donnons aux formateurs dans ces grands projets technopédagogiques institutionnels et des limites d'orienter et de centrer les dispositifs de formation continue sur l'adaptation des formateurs aux dispositifs implantés.

La littérature sur la conception collaborative (Borko, 2004; Parke et Coble, 1997; Voogt et al., 2015) ou la conception participative (Muller et Kuhn, 1993) de dispositifs pédagogiques renouvelle le rôle des formateurs; d'utilisateur des dispositifs, ils deviennent des co-concepteurs. Ce processus permet aux formateurs d'adapter le matériel ou le dispositif existant à leur contexte en se confrontant, en collaboration avec les conseillers/ingénieurs pédagogiques, aux particularités des dispositifs technopédagogiques 
(Voogt et al., 2015). Ce renouvellement du rôle des formateurs rejoint la volonté des participants à notre enquête de retrouver dans leur environnement d'enseignement une réponse à leurs besoins et laisse à penser que ces environnements d'enseignement peuvent non seulement servir d'espace d'enseignement et d'apprentissage, mais également d'occasions de formation continue.

\section{Conclusion}

L'analyse des résultats de l'enquête que nous avons réalisée montre que les formateurs semblent préférer davantage l'autoformation et l'accompagnement personnalisé, que la possibilité de participer à une formation de type magistral. Conséquemment, l'enquête fait ressortir le rôle-clé que peuvent jouer les techniciens dans l'usage de ces nouveaux dispositifs technopédagogiques et le rôle déterminant d'une combinaison de ressources afin de soutenir les formateurs au quotidien dans leur appropriation de ces dispositifs. Ainsi, en plus de la présence et de l'accessibilité d'un technicien ou d'un accompagnateur en cours d'utilisation des dispositifs, les répondants suggèrent de combiner d'autres ressources, dont des tutoriels, des guides ou des documents explicatifs.

Outre l'intérêt de poursuivre les recherches sur l'usage des dispositifs technopédagogiques de type visioconférence, l'analyse des résultats nous a menés à des constats qui nous apparaissent interreliés. D'un côté, ces résultats pointent vers une adaptation des environnements d'enseignement afin que les formateurs puissent y trouver une réponse à leurs besoins en termes de formation, d'information ou de ressources. Selon les résultats de notre recherche, ils veulent être soutenus le plus possible au niveau de leur activité d'enseignement. Dans cette perspective, le rôle des conseillers/ingénieurs pédagogique est moins de développer des formations, que de réfléchir à comment soutenir les formateurs dans leur appropriation des transformations de leur environnement d'enseignement (Meyer et al., 2016). D'un autre côté, l'importance accordée au développement des compétences technologiques par les formateurs nous a amenés à nous poser la question du rôle donné à ces derniers dans les démarches institutionnelles d'implantation de nouveaux dispositifs technopédagogiques. II nous apparaît que le rôle " d'acteurs passifs » qui leur est généralement conféré les amène à vouloir pallier leur manque de connaissances technologiques et, de ce fait, à chercher à s'adapter aux nouveaux équipements. Au final, il nous semble important de garder à l'esprit que c'est l'ensemble du milieu social qui est concerné par une innovation (Alter, 2010) et que l'usage des nouveaux dispositifs technopédagogiques requiert de considérer également l'innovation pédagogique au niveau organisationnel (Albero et al, 2008; Bédard et Béchard, 2009). Ce faisant, les présents résultats portent à croire qu'un rapprochement doit être réalisé, au niveau organisationnel, entre les actions et les décisions menant à l'implantation de nouveaux dispositifs technopédagogiques et celles destinées à la formation continue des formateurs. Ainsi, le fait de concevoir les formateurs comme des co-concepteurs de ces dispositifs permettrait, à l'instar des travaux de Poumay (2014), de leur offrir plusieurs portes d'entrée à l'innovation et non de les contraindre à la nécessité de s'adapter à des changements imposés.

\section{Liste de références}

Albero, B. (2000). L'autoformation en contexte institutionnel : du paradigme de l'instruction au paradigme de l'autonomie. L'Harmattan.

Albero, B. (2004). Technologies et formation; travaux, interrogations et pistes de réflexion dans un champ de recherché éclaté. Savoirs, 5(2), 9-69.

Albero, B. (2011). Le couplage entre pédagogie et technologies à l'université: cultures d'action et paradigmes de recherche. Revue internationale des technologies en pédagogie universitaire, 8(1-2), 11-21. 
Albero, B. (2014). La pédagogie à l'université entre numérisation et massification. Apports et risques d'une mutation. Dans G. Lameul et C. Loisy (dir.), La pédagogie universitaire à l'heure du numérique. Questionnement et éclairage de la recherche (p. 27-53). De Boeck Supérieur.

Albero, B., Linard, M. et Robin, J. Y. (2008). Petite fabrique de l'innovation à l'université : quatre parcours de pionniers. L'Harmattan.

Alhlak, B. A., Ramakrisnan, P., Hameed, Z. S. et Mohseni, H. R. (2012). Video conference: integrated tool for identifying CSF in education development in UiTM. Procedia-Social and Behavioral Sciences, 2012(67), 102-113.

Alter, N. (2010). L'innovation ordinaire. Presses universitaires de France.

Bachy, S. (2014). Un modèle-outil pour représenter le savoir technopédagogique disciplinaire des enseignants. Revue internationale de pédagogie de l'enseignement supérieur, 30(2), mis en ligne le 10 mai 2014. https://journals.openedition.org/ripes/821

Baron, G.-L. et Bruillard, E. (1996). L'informatique et ses usagers dans l'éducation. Presses. Universitaires de France.

Bates, T., Johnson, N., Donovan, T., Seaman, J., Mayer, D., Martel, É., Paul, R., Desbiens, B., Forssman V. et Poulin, R. (2019). Évolution de la formation à distance et de l'apprentissage en ligne dans les universités et collèges du Canada. Rapport public. Association canadienne de recherche sur la formation en ligne. Toronto.

Bédard, B. (2006). Enseigner autrement, oui mais pourquoi et comment? Le cas d'un cours universitaire de premier cycle. Dans N. Rege Colet et M. Romainville (dir.), La pratique enseignante en mutation à l'université (p. 83-101). De Boeck.

Bédard, D. et Béchard, J.-P. (dir.) (2009). Innover dans l'enseignement supérieur. Presses Universitaires de France.

Bélisle, M., Lison, C. et Bédard, D. (2016). Accompagner le scholarship of teaching and learning. Dans A. Daele et E. Sylvestre (dir.), Comment développer le conseil pédagogique dans l'enseignement supérieur?: conseillers pédagogiques, enseignants, formateurs (p. 75-90). De Boeck Supérieur.

Borko, H. (2004). Professional development and teacher learning: Mapping the terrain. Educational researcher, 33(8), 3-15.

Cuban, L. (1986). Teachers and machines: The classroom use of technology since 1920. Teachers college press.

De Ketele, J-M. (2010). La pédagogie universitaire : un courant en plein développement. Revue française de pédagogie, 2010(172), 5-13.

Glover, I., Hepplestone, S., Parkin, H., Rodger, H. et Irwin, B. (2016). Pedagogy first: realising technology enhanced learning by focusing on teaching practice. British journal of educational technology, 47(5), 993-1002.

Gillies, D. (2008). Student perspectives on videoconferencing in teacher education at a distance. Distance education, 29(1), $107-118$.

Goastellec, G. (2014). Les mutations de l'enseignement supérieur en Europe. Comprendre les transformations à l'œuvre. Dans G. Lameul et C. Loisy (dir.), La pédagogie universitaire à l'heure du numérique. Questionnement et éclairage de la recherche (p. 55-68). De Boeck Supérieur.

Hativa, N. (2000). Teaching for effective learning in higher education. Kluwer academic publisher.

Jacquinot, G. (1985). L'école devant les écrans. ESF.

Jacquinot, G. et Fichez, E. (2008). L'université et les TIC. Chronique d'une innovation annoncée. De Boeck.

Jézégou, A. (2010). Créer de la présence à distance en e-learning: cadre théorique, définition et dimensions clés. Distance et savoirs, 8, 257-274.

Kukulska-Hulme, A. (2012). How should the higher education work force adapt to advancements in technology for teaching and learning?. The internet and higher education, 15(4), 247-254.

Lameul, G. et Loisy, C. (2014). La pédagogie universitaire à l'heure du numérique. De Boeck

Lawson, T., Comber, C., Gage, J. et,Cullum-Hanshaw, A. (2010) Images of the future for education? Videoconferencing: a literature review. Technology, pedagogy and education, 19(3), 295-314.

Linard, M. (1996). Des machines et des hommes. Apprendre avec les nouvelles technologies. (2e.éd.). L'Harmattan.

Lison, C. et Jutras, F. (2014). Innover à l'université : penser les situations d'enseignement pour soutenir l'apprentissage, Revue internationale de pédagogie de l'enseignement supérieur, 30(1), mis en ligne le 07 avril 2014. http://ripes.revues.org/769

Macedo-Rouet, M. (2009). La visioconférence dans l'enseignement. Distances et savoirs, 7(1), 65-91.

Meyer, F., Lameul, G., Petit, M., Proust, S., Bédard, D. et Nocéra Picand, C. (2016). La recherche orientée par la conception, une voie pour soutenir le développement professionnel des conseillers pédagogiques: le cas du projet TOPIC. Communication présentée dans le cadre du symposium "Regards croisés sur la formation des formateurs en enseignement supérieur à l'ère du numérique », Julie Lyne Leroux, Florian Meyer et Jean-Marc Nolla. 84e congrès de l'Association francophone pour le savoir (ACFAS), Montréal, 10 mai.

Mishra, P., et Koehler, M. J. (2006). Technological pedagogical content knowledge: A framework for teacher knowledge. Teachers college record, 108(6), 1017-1054. 
Muller, M. J. et Kuhn, S. (1993). Special issue on participatory design. Communications of the ACM, 36(6), 24-28.

Murthy, S., lyer, S. et Warriem, J. (2015). ET4ET: A large-scale faculty professional development program on effective integration of educational technology. Educational technology and society, 18(3), 16-28.

O'Donnell, C., Masson, A., Ross, V., Virapen, K. et Harrison, J. (2012). Encouraging creative and innovative 'viewpoints' in curriculum design. Dans D. Kelsey (dir.), Enhancing learning and teaching in higher education in Northern Ireland (p.18-23). The Higher education academy, Irlande du Nord.

Paillé, P. et Mucchielli, A. (2016). L'analyse qualitative en sciences humaines et sociales. (4e éd.). Armand Colin.

Parke, H. M. et Coble, C. R. (1997). Teachers designing curriculum as professional development: A model for transformational science teaching. Journal of research in science teaching, 34(8), 773-789.

Pires, A. (1997). Échantillonnage et recherche qualitative: essai théorique et méthodologique Dans J. Poupart, J. P. Deslauriers, L. H. Groulx, A. Laperrière, P. Mayer et A. Pires (dir.), La recherche qualitative. Enjeux épistémologiques et méthodologiques (p. 113-169). Gaëtan Morin, Éditeur.

Poellhuber, B., Racette, N. et Chirchi, M. (2012). De la présence dans la distance par la visioconférence Web. Revue internationale des technologies en pédagogie universitaire, 9(1-2), 63-77.

Poumay, M. (2014). L'innovation pédagogique dans le contexte de l'enseignement supérieur. Dans G. Lameul et C. Loisy (dir.), La pédagogie universitaire à l'heure du numérique. Questionnement et éclairage de la recherche (p. 27-53). De Boeck Supérieur.

Romainville, M. (2006). Introduction. Dans N. Rege Colet et M, Romainville (dir.), La pratique enseignante en mutation à l'université (p. 7-13). De Boeck.

Shin, N. (2002). Beyond interaction: The relational construct of "transactional presence". Open learning, 17(2), 121-137.

Shulman, L. (1986). Those who understand: Knowledge growth in teaching. Educational researcher, 15(2), 4-14.

Shulman, L. S. (1987). Knowledge and teaching: Foundations of the new reform. Harvard educational review, 57(1), 1-23.

Thibault, F. (2007). Enjeux de l'enseignement à distance pour l'université française 1947-2004 (thèse de doctorat, Université Paris-Nord - Paris XIII). http://tel.archives-ouvertes.fr

Viberg, O., Bälter, O., Hedin, B., Riese, E. et Mavroudi, A. (2018). Faculty pedagogical developers as enablers of technologyenhanced learning. British journal of educational technology, 50(5), 1-14.

Villeneuve, S. (2011). L'évaluation de la compétence professionnelle des futurs maîtres du Québec à intégrer les technologies de l'information et des communications (TIC) : maîtrise et usages. (thèse de doctorat, Université de Montréal, Canada). https://papyrus.bib.umontreal.ca/xmlui/bitstream/handle/1866/6057/Villeneuve Stephane 2011 these.pdf

Voogt, J., Laferrière, T., Breuleux, A., Itow, R. C., Hickey, D. T. et McKenney, S. (2015). Collaborative design as a form of professional development. Instructional science, 43(2), 259-282.

Wallet, J. (2007). Le Campus numérique FORSE; analyses et témoignages. Publications des Universités de Rouen et du Havre. 